\title{
Are You Being Rejected or Excluded? Insights from Neuroimaging Studies Using Different Rejection Paradigms
}

\author{
Preethi Premkumar \\ Division of Psychology, School of Social Sciences, Nottingham Trent University, Nottingham, United Kingdom
}

\begin{abstract}
Rejection sensitivity is the heightened tendency to perceive or anxiously expect disengagement from others during social interaction. There has been a recent wave of neuroimaging studies of rejection. The aim of the current review was to determine key brain regions involved in social rejection by selectively reviewing neuroimaging studies that employed one of three paradigms of social rejection, namely social exclusion during a ball-tossing game, evaluating feedback about preference from peers and viewing scenes depicting rejection during social interaction. Across the different paradigms of social rejection, there was concordance in regions for experiencing rejection, namely dorsal anterior cingulate cortex (ACC), subgenual ACC and ventral ACC. Functional dissociation between the regions for experiencing rejection and those for emotion regulation, namely medial prefrontal cortex, ventrolateral prefrontal cortex (VLPFC) and ventral striatum, was evident in the positive association between social distress and regions for experiencing rejection and the inverse association between social distress and the emotion regulation regions. The paradigms of social exclusion and scenes depicting rejection in social interaction were more adept at evoking rejection-specific neural responses. These responses were varyingly influenced by the amount of social distress during the task, social support received, self-esteem and social competence. Presenting rejection cues as scenes of people in social interaction showed high rejection sensitive or schizotypal individuals to under-activate the dorsal ACC and VLPFC, suggesting that such individuals who perceive rejection cues in others down-regulate their response to the perceived rejection by distancing themselves from the scene.
\end{abstract}

KEY WORDS: Social distance; Gyrus cinguli; Prefrontal cortex; Social support.

\section{INTRODUCTION}

The concept of social rejection has been widely researched since the 1960s. Early theoretical constructs of social rejection were built on theories of approach and avoidance and social learning. ${ }^{1-3)}$ One of the earliest models of response to rejection took the form of interpersonal choice and expectancy of outcome in the context of achievement of one's goals. ${ }^{1)}$ Here, a need for affiliation is experienced as a concern by the individual over positive affective relationships, while its converse is a fear of rejection. A similar prototype developed by Mehrabian and Ksionzky ${ }^{2)}$ regarded rejection sensitivity as being on a continuum with need for affiliation, wherein there is a general expectation of positive reinforcement (need for af-

\footnotetext{
Received: July 20, 2012 / Revised: August 27, 2012

Accepted: August 28, 2012

Address for correspondence: Preethi Premkumar, PhD

Division of Psychology, Nottingham Trent University, Burton Street, Nottingham NG1 4BU, United Kingdom

Tel: +44-(0)115-848-4511, Fax: +44-(0)115-848-2390

E-mail: Preethi.premkumar@ntu.ac.uk
}

filiation) or negative expectation (rejection sensitivity) from others. A more recent cognitive explanation for rejection by peers in children is having a poor theory of mind. ${ }^{4)}$ However, this relation is not straightforward and mediated by factors such as poor family relations and amount of prosocial behaviour. Caputi et al. ${ }^{4)}$ found that having a better theory of mind at 5 years of age was associated with having better prosocial skills at six years and in turn with greater likelihood of being nominated as being most-liked by peers at seven years, whereas having poorer prosocial skills at five years was associated with a greater likelihood of being nominated as being least-liked by peers at six and seven years. These rejection experiences emphasize peer relations.

Rejection sensitivity has also been studied in terms of close relationships. Downey and Feldman ${ }^{3)}$ described rejection sensitivity as a tendency to 'anxiously expect, readily perceive, and overreact to rejection'. In romantic relationships, such individuals tend to expect harmful intent from their partner towards them and experience more insecurity and less satisfaction with their relationship, ir-

(c) This is an Open-Access article distributed under the terms of the Creative Commons Attribution Non-Commercial License (http://creativecommons.org/licenses/by-nc/3.0) which permits unrestricted non-commercial use, distribution, and reproduction in any medium, provided the original work is properly cited 
respective of whether or not the partner feels satisfied with the relationship. ${ }^{3)}$ The expression-based authenticity doubts model ${ }^{5)}$ describes the situation where individuals who are insecure about their romantic relationship are likely to perceive themselves as expressing more vulnerability. In fact, people do respond with more caution and feign positivity towards individuals who express interpersonal sensitivity. ${ }^{5)}$ In turn, participants who had expressed more vulnerability towards their partner were also more likely to experience more authenticity doubts about their partner because they felt their partner regarded them as vulnerable.

\section{Neural Effects of Social Rejection}

There has been a recent surge in research into the neural effects of social rejection. ${ }^{6}$ ) One of the key neural substrates for perceiving social rejection is the anterior cingulate cortex (ACC). Conflict detection and error monitoring tasks engage the ACC. ${ }^{7)}$ Reward processing also recruits the ACC; here the ACC is involved in updating and integrating information on the reward value of a given event and predicting future outcome. ${ }^{8)}$ It is also involved in the experience of physical pain. ${ }^{6)}$ A combination of these roles may account for ACC activation during rejection tasks. There are methodological challenges in neuroimaging the response to social rejection, the chief challenge being simulating the direct rejection experience. As rejection is an interpersonal emotion expressed both verbally $^{9,10)}$ and non-verbally, ${ }^{2,5)}$ task designs need to engage the person in social interaction. Neuroimaging studies have employed a range of paradigms to study different types of perceived social rejection, the most prominent being rejection as peer exclusion. In comparison, studies on neural effects of rejection from close relatives are rare. It is likely that the degree of closeness of the third person(s) is an important variable in explaining variability in the neural response to rejection.

The aim of the article was to determine the key brain areas for social rejection. A selective review of studies on the neural effects of social rejection was conducted, taking into account the level of proximity of the interpersonal interaction being implied. A further aim of the review was to examine in each study how the neural response to experimental manipulations of social rejection during fMRI related to scores on behavioural measures in order to understand the emotional and social significance of the neural responses. A more comprehensive review ${ }^{6}$ explores the neurological and evolutionary significance of the fMRI findings of response to social rejection. Due to the large number of neuroimaging studies examining rejection under different contexts (social exclusion, $n=20$; social evaluation, $\mathrm{n}=6$; and rejection cues as words and scenes, $n=8$ ), a selection of studies from each experimental paradigm has been reviewed.

\section{Task Design}

Rejection related tasks have frequently involved deception or manipulation of a situation by confederates, the most widely used task being "Cyberball". In the original version as presented in the study by Eisenberger et al., ${ }^{11}$ ) participants played with two other virtual players, while participants were made to believe that they were playing with real players. In the first part of the task, participants were told that they could not take part in the game due to technical difficulties (implicit social exclusion condition, ISE). Next, participants engaged in a ball-tossing game (social inclusion) and in the third part participants were included in the game for a while, but then excluded (explicit social exclusion condition, ESE). This type of social exclusion seems to draw on theories of social identity (in-group vs. out-group status) ${ }^{11)}$ and the need for affiliation with and approval by peers. i.e., it should be 'This type of social exclusion seems to draw on theories of social identity (in-group vs. out-group status) ${ }^{12)}$ and the need for affiliation with and approval by peers.' Such approval is achieved on a competitive basis, such as being rated as most-liked by others. ${ }^{13)}$

Social preference - receiving feedback about being liked or disliked by peers - is another form of acceptance or rejection that has been applied to fMRI study design. The social evaluation task ${ }^{13)}$ is used to assess the neural response to receiving feedback from others. Here, participants viewed pictures of others while deciding whether they like the pictured person. Next, participants received feedback about whether the persons pictured in the task liked the participant or not, although in truth no such feedback was actually provided by the pictured persons and these responses were randomly set by the experimenter. This type of rejection has been studied behaviourally previously. ${ }^{1)}$ Eighty-five high school boys were put into groups of $\mathrm{n}=6$ and asked to perform cognitive skills tests. ${ }^{1)}$ Participants were given a false performance score of $48 \%$, but were led to believe that the other group members had different (higher and lower) scores to them. Participants then gave preference ratings of the other group members and received feedback about the other members' preference of the person. Again, this feedback was pre-determined. Participants then indicated their single most pre- 
ferred other based on this feedback. Findings suggested that participants were more likely to rate the other group members as being available to them if they had similar performance scores than if the other group members had higher performance scores. Participants also rated those with lower performance scores than their own as being less available possibly because of the perception that the other group members would be less likely to see themselves as being accepted by the participant. However, participants were more likely to rate their own availability to the other group members as higher if the other group members had higher performance scores. This study shows how people's preference for others is influenced by their own perceived ability. Children who were rejected from a hypothetical in-group were more likely to express indirect aggression (e.g., ignoring, gossiping about, deceiving, rejecting, or excluding another) towards in-group and out-group members compared to children who were accepted by the in-group. ${ }^{14)}$

Rejection cues have also been presented as words and pictures in ways that suggest on-going social interaction. ${ }^{15,16)}$ Here, no assumptions are made about the nature of the participant's own involvement in the social interaction. Instead, the stimuli act as prompts for an imagined emotional context with which the participant may have familiarity. The assumption here is that if participants find the cue arousing, it indicates the participant's greater the familiarity with the imagined situation.

\section{FMRI BOLD RESPONSE TO REJECTION AS SOCIAL EXCLUSION DURING 'CYBERBALL'}

Eisenberger et al. ${ }^{11)}$ likened the response to rejection to physical pain. In their study, 13 undergraduates played Cyberball. The dorsal ACC (dACC), right insula and right ventral prefrontal cortex (PFC) were activated more during ESE than inclusion. Greater dACC activation and lower ventral PFC activation in turn were correlated with greater self-reported distress during ESE. The authors inferred that dACC involvement reflected greater arousal to exclusion than inclusion conditions, because of the correlation between ACC activation and self-reported distress due to exclusion. However, the study did not directly compare neural response to the implicit versus explicit social exclusion conditions. This comparison would have been useful in determining neural activation differences between internal attribution (ESE) and external attribution of social exclusion (ISE). ${ }^{10)}$

Next, Eisenberger et $_{\text {al. }}{ }^{17)}$ examined the mediating role of the neural response to exclusion between the amount of daily social support received and the cortisol stress response to a stress-provoking exercise. Thirty-two participants (mean age 20 years) first took part in a daily social interactions task where over the course of 10 days they recorded the amount of support they felt they received during any social interaction taking place at five random times during the day. Participants then performed the Cyberball task while undergoing fMRI and reported level of social distress during the inclusion and exclusion conditions. Finally, participants performed a stress test where they were given five minutes to prepare a speech to be delivered to two judges and to perform an arithmetic task under observation (counting backwards in 13 seconds from starting from the number 2,083). Salivary cortisol levels were measured before and after the stress test. Although the neural response in the $\mathrm{AACC}$ and ventral PFC to exclusion found previously ${ }^{11)}$ was not replicated, social distress was positively associated with dACC activation and inversely associated with right ventral PFC during exclusion compared to inclusion. In addition, greater social support was associated with lower right dACC and left superior frontal gyrus (Brodmann Area, BA 8) activity during exclusion, activations that were in turn positively associated with cortisol change following stress. Mediation analyses suggested dACC and BA 8 activity mediated the association between social support and cortisol level. Finally, there was positive connectivity between $\mathrm{AACC}$ and BA8 activation and hypothalamus activation arising from the correlation with cortisol level. These findings show how in an experimental simulation of social interaction leading to exclusion, neural activity in predictable brain areas for rejection can meaningfully relate to real-world social interaction and performance-related stress, although the use of experience sampling to measure social interaction needs to be validated with other social support self-report measures.

Onoda et $_{\text {al }}{ }^{18)}$ did study the neural effect of social support on social exclusion. In addition to the standard inclusion and exclusion blocks, three exclusion blocks were presented accompanied by statements indicating social support, e.g., "Sorry, I know it was unpleasant for you to be excluded" or "I also found the task unpleasant when I was a participant". Participants (26 undergraduate students) provided distress ratings at the end of scanning reflecting on their experience during exclusion compared to inclusion. The right posterior cingulate cortex and insula were activated more during social exclusion than inclusion and left somatosensory area during inclusion than 
exclusion. During exclusion compared to exclusion with emotional support, several areas including the right dorsolateral PFC (DLPFC), right ventrolateral PFC (VLPFC) and right insula were activated more strongly. During exclusion with emotional support compared to exclusion alone, the medial PFC, left VLPFC, bilateral temporal pole, left superior temporal sulcus and left precuneus were activated more strongly. During exclusion compared to inclusion, greater self-reported pain correlated with greater dACC and ventral ACC (vACC) activity. During exclusion with emotional support compared to exclusion alone, decreased self-reported pain correlated positively with decreased vACC activity. In turn, functional connectivity between greater vACC activity and lesser activity in left DLPFC, postcentral gyrus, posterior cingulate cortex, visual cortex and cerebellum during exclusion than exclusion with social support was observed. This study provides further evidence for the role of social support in rejection experience. These findings may suggest that the amount of perceived support modulates the experience of rejection in the $\mathrm{AACC}$ and $\mathrm{VACC}$ by reducing distress, whereas the VLPFC and medial PFC may be involved in implicit emotion regulation or reappraisal of exclusion under different levels of support. Whether this regulatory function of the VLPFC and medial PFC directly impacts on distress due to rejection in the $\mathrm{AACC}$ and $\mathrm{vACC}$ is not known, as functional connectivity was not found between these regions.

However categorising the participants as having low or high self-esteem ${ }^{19)}$ did reveal inverse function connectivity between the two sets of brain regions as a function of self-esteem. Compared to the high self-esteem group, the low self-esteem group showed greater activation of dACC and vACC, as well as other regions such as right insula, right hippocampus, bilateral parahippocampus and right cerebellum. Importantly, inverse functional connectivity was found between $\mathrm{AACC}$ and right VLPFC and right medial PFC, such that in the low self-esteem group, greater dACC activation during exclusion was associated with greater right VLPFC and right medial PFC activation, while in the high self-esteem group, greater dACC activation was associated with lower right VLPFC and right medial PFC activation. These findings suggest that VLPFC and medial PFC are involved in emotion regulation in terms of reducing dACC activation due to rejection in high self-esteem individuals, but such regulation is altered in individuals with low self-esteem.

Masten et $a .^{20)}$ investigated the role of social competence and rejection sensitivity in the neural response to social exclusion. One view is that individuals high in social competence would have better peer relationships and therefore less concern about rejection. An opposing view is that those high in social competence would have a greater need to maintain their social status and therefore be more concerned about being rejected. Adolescent participants $(n=23$, mean age $=13$ years) played Cyberball under the belief that they were playing with real people. During exclusion compared to inclusion, participants activated the right subgenual ACC, right VLPFC, right ventral striatum and right insula. Furthermore, distress correlated positively with activity in the subgenual ACC, insula, anterolateral PFC in the left hemisphere and in one region in the right VLPFC during exclusion. Distress also correlated negatively with activity in the right hemisphere of the ventral striatum, two VLPFC regions and dorsomedial PFC. Right subgenual ACC and insula activation correlated inversely with right hemisphere activation in the ventral striatum, VLPFC and dorsomedial PFC. Greater rejection sensitivity correlated positively with greater activity in the right dACC, left anterolateral PFC and left precuneus during exclusion relative to inclusion, while greater social competence correlated positively with activity several regions, including left dACC and bilaterally in the insula, VLPFC and ventral striatum. The findings of a negative association between distress and right VLPFC and ventral striatum signified greater modulation of affect in these regions during exclusion. Positive associations between subgeunal ACC activity and distress, and ACC activity and rejection sensitivity and social competence indicated more intense experience of rejection during exclusion in these regions.

Sebastian et al. ${ }^{21)}$ modified the Cyberball task to pseudo-randomise the inclusion and exclusion blocks in order to minimize task order effects and task habituation. In addition in order to balance motor responses during the inclusion and exclusion conditions, participants (adolescents, $n=19$, mean age $=15.44$ years; and adults, $n=16$, mean age $=28.7$ years) were asked to provide button-clicks for throws between the other two players. Participants then rated their social distress on recollection of the inclusion and exclusion experiences and resistance to peer influence. Adolescents reported being more distressed and excluded during the exclusion, than inclusion, condition and had weaker resistance to peer influence compared to adults. Greater activity during exclusion than inclusion was observed in two predicted areas, namely right subgenual ACC and left VLPFC in addition to several other unpredicted areas. The condition-by-age group inter- 
action in the right VLPFC revealed greater activation during exclusion than inclusion in adults, but weaker during exclusion than inclusion in adolescents, a finding that is resonant with the view that the right VLPFC is involved in emotion regulation by virtue of its inverse association with distress during exclusion. ${ }^{20)}$ The findings stress the neurodevelopmental differences between adults and adolescents. The VLPFC may be less adept at emotion regulation in adolescents when coupled with greater distress and weaker resistance to peer influence. Contrastingly, the left medial PFC was more prone to influence by resistance to peer influence scores in adolescents, such that weaker resistance to peer influence predicted greater left medial PFC activation in adolescents, but not in adults.

These findings clearly reveal prime brain regions for experiencing rejection, namely $\mathrm{dACC}$, subgenual ACC and $\mathrm{VACC}$ and brain regions for emotion regulation contingent on rejection, namely medial PFC and VLPFC and ventral striatum. Table 1 presents the Montreal Neurological Institute (MNI) coordinates of activation or deactivation clusters observed in each study during comparison of different rejection task conditions. Fig. 1 depicts the averaged MNI coordinates across studies on a two-dimensional space, where the size of the ring represents the relative variability between studies in the y-coordinate. The functional dissociations of these two sets of brain regions are substantiated somewhat by their positive and inverse associations respectively with social distress, rejection sensitivity, while VLPFC activation to exclusion has positive association with social support, social competence and self-esteem, although it must be acknowledged that these findings are not consistent across studies. Table 2

Table 1. MNI coordinates of activation or deactivation clusters observed in each study during comparison of different rejection task conditions

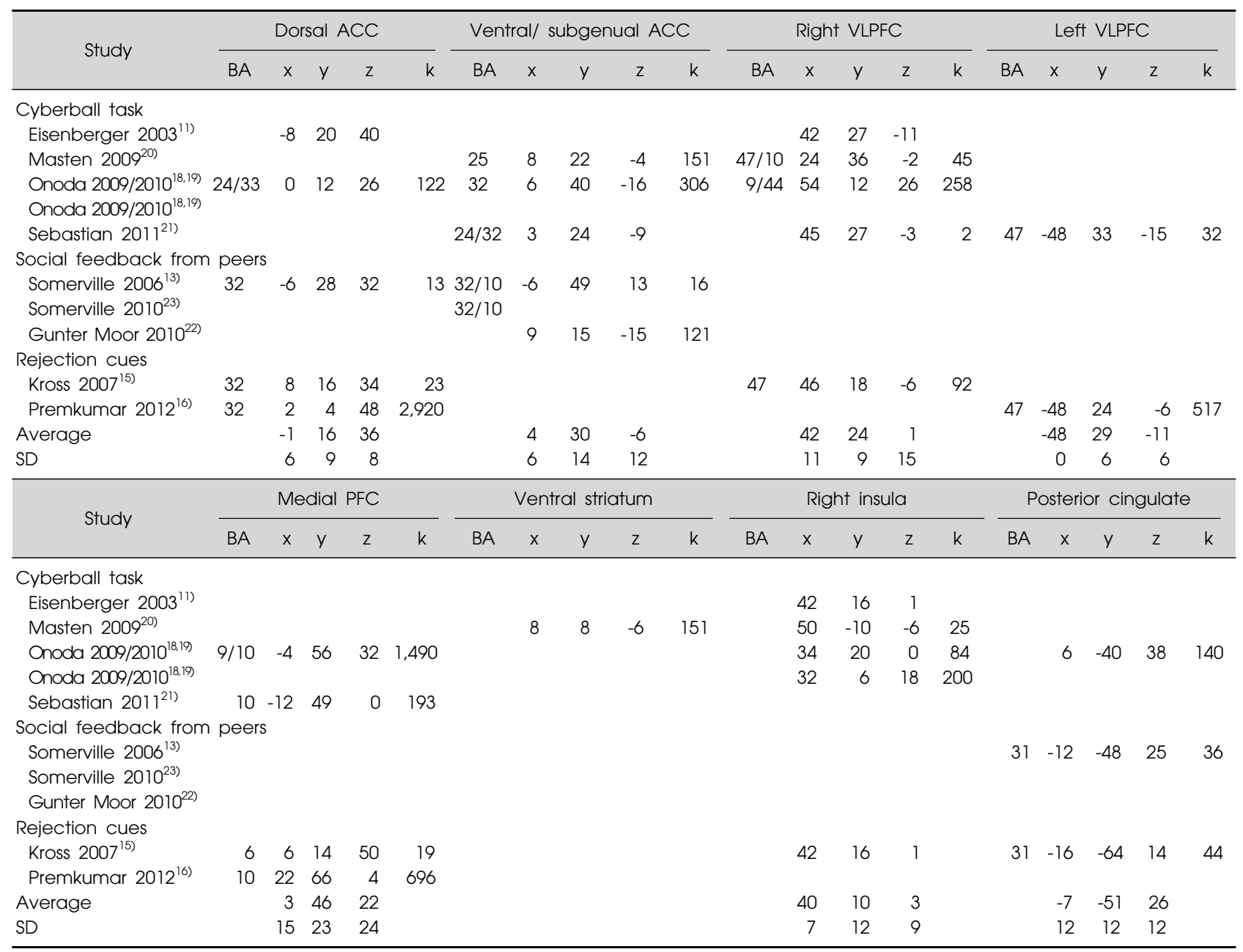

MNI, Montreal Neurological Institute; ACC, anterior cingulate cortex; BA, Brodmann area; PFC, prefrontal cortex; k, cluster size; VLPFC, ventrolateral prefrontal cortex; $x, y, z, M N I$ coordinates; SD, standard deviation. 

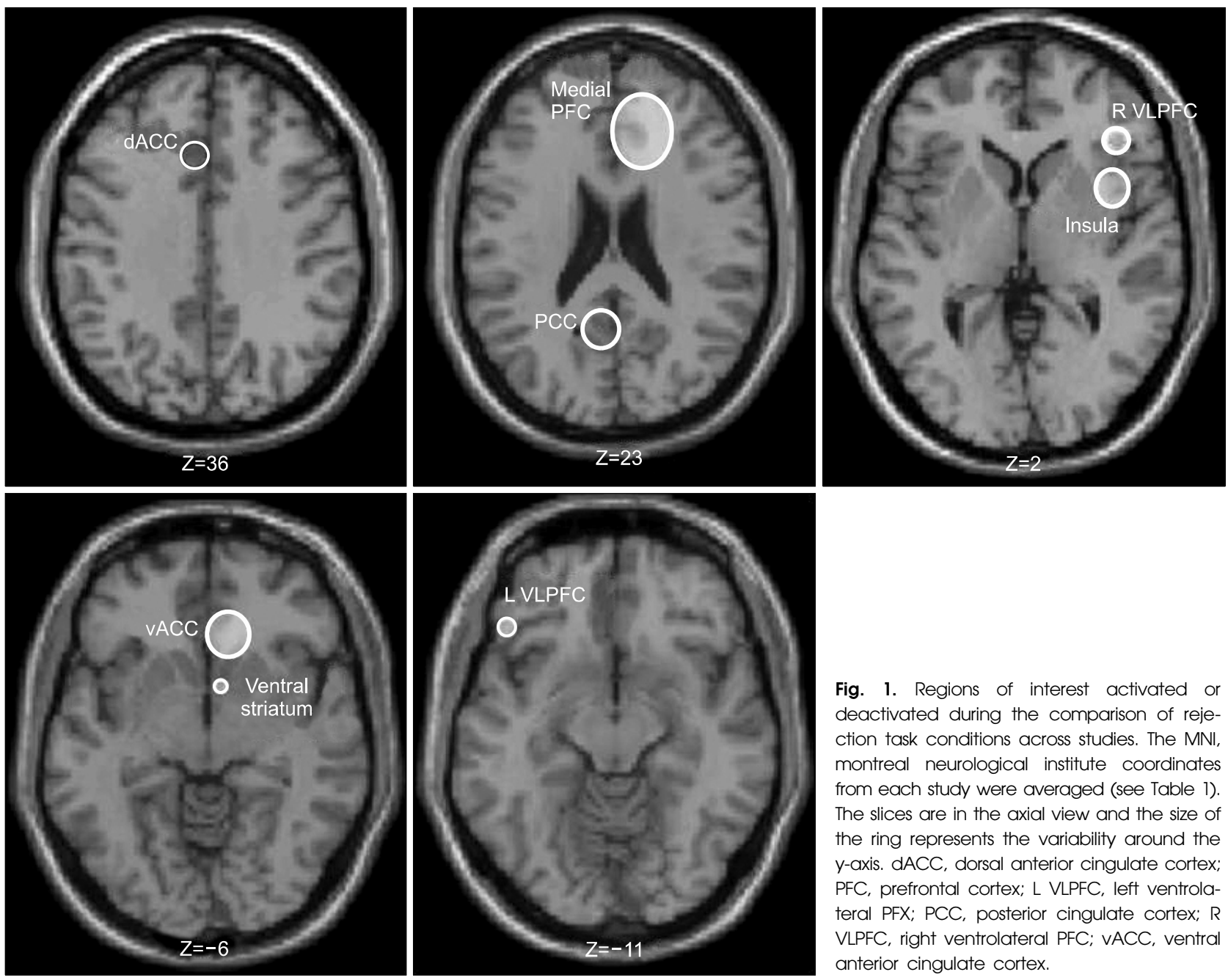

Fig. 1. Regions of interest activated or deactivated during the comparison of rejection task conditions across studies. The MNI, montreal neurological institute coordinates from each study were averaged (see Table 1). The slices are in the axial view and the size of the ring represents the variability around the $y$-axis. $A A C C$, dorsal anterior cingulate cortex; PFC, prefrontal cortex; L VLPFC, left ventrolateral PFX; PCC, posterior cingulate cortex; $\mathrm{R}$ VLPFC, right ventrolateral PFC; VACC, ventral anterior cingulate cortex.

presents the MNI coordinates of activation or deactivation clusters that are correlated with different self-report measures. Fig. 2 depicts the averaged MNI coordinates, where the size of the ring represents the relative variability between studies in the y-coordinate.

\section{REJECTION AS NEGATIVE SOCIAL EVALUATION FROM PEERS}

Somerville et al. ${ }^{13)}$ employed the social evaluation task to study the neural response to peer acceptance or rejection by asking participants to view pictures of others while deciding whether they liked them or not. The participant was led to believe that the people pictured in the task had chosen whether they liked the participant or not, although no such feedback was received from the others; these responses were randomly set by the experimenter. In a further experiment, participants were asked to judge whether the people pictured in the task would like them and received feedback about whether the pictured people liked them. In both experiments, the dACC was deactivated more when feedback from the other person about the participant was consistent with the participant's judgement of that person (congruent) than when the feedback received was inconsistent with the participant's view of the person (incongruent). The left subgenual ACC/medial PFC was activated more when feedback was positive, i.e., the other person liked the participant, than when negative, i.e., the other person did not like the participant, regardless of whether the participant indicated that they liked the person or did not like the person. The findings illustrated differential involvement of dorsal and subgenual ACC; the dorsal ACC's involvement suggests error monitoring, rather than semantic evaluation, since trials where the participant expressed dislike, but the feedback suggested acceptance also engaged the dorsal ACC. Whereas the sub- 
Table 2. MNI coordinates of activation or deactivation clusters that are correlated with different self-report measures

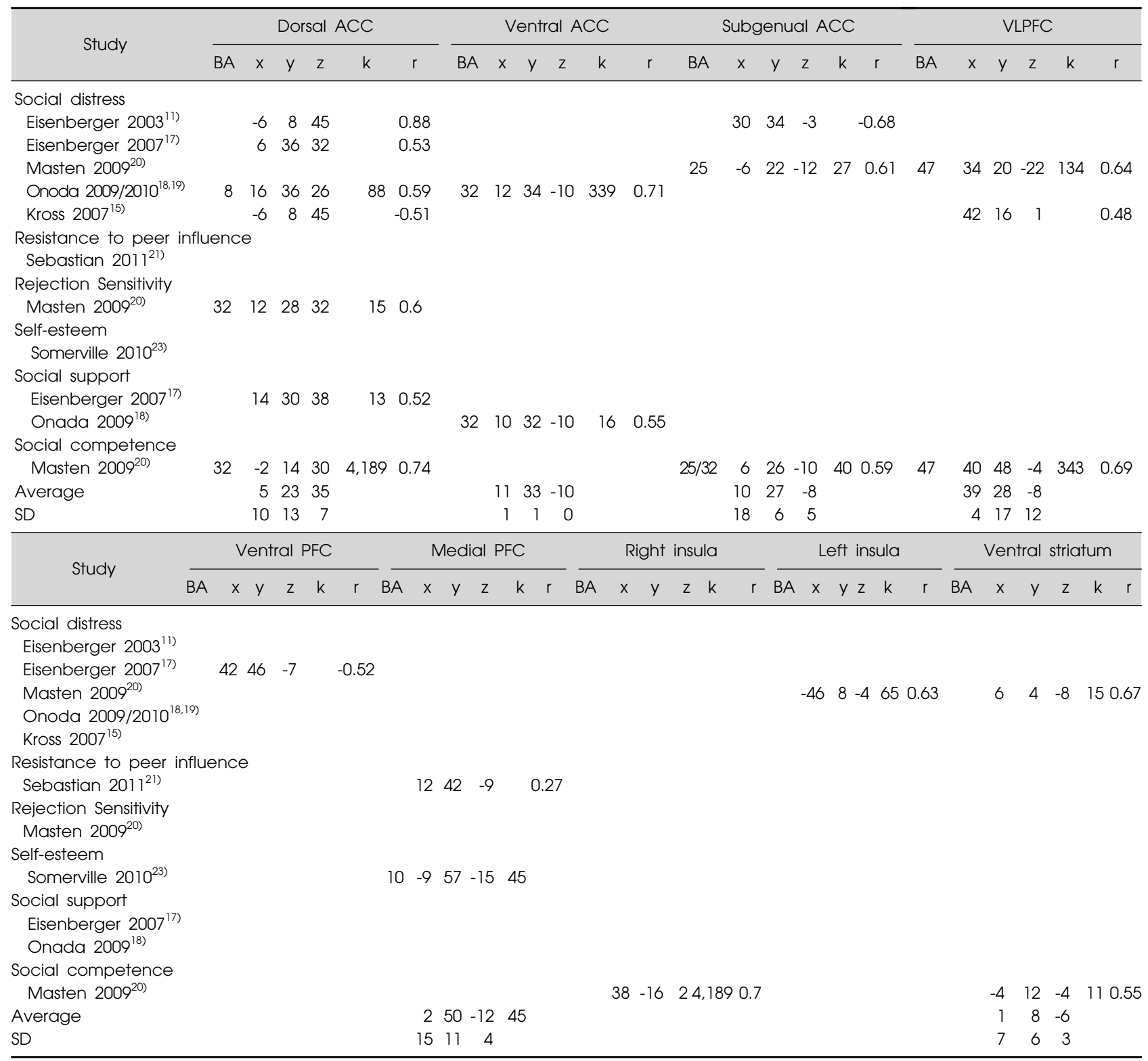

MNI, Montreal Neurological Institute; ACC, anterior cingulate cortex; BA, Brodmann area; PFC, prefrontal cortex; $k$, cluster size; r, correlation coefficient; VLPFC, ventrolateral prefrontal cortex; $x, y, z, M N I$ coordinates; SD, standard deviation.

genual ACC seems to attend to feedback valence without integrating information about how the participant judged the other. However, how information concerning rejection is encoded was not examined, i.e., where the participant expresses liking, but the feedback received is dislike. Participants may show preferences for others and decide others' preference for them based on their own beliefs about their attractiveness, but may experience more rejection when such preferences are met with negative feedback. As Rosenfeld ${ }^{1)}$ had observed, individuals with a greater fear of rejection tend to choose peers with similar cognitive abilities than those with higher cognitive abilities.

Gunther Moor et $a l^{22)}$ were interested specifically in neural changes when expectancy about being liked or disliked was followed by peer feedback that suggested acceptance or rejection, i.e., the comparison between congruent and incongruent conditions, as feelings of social rejection are likely to be more intense when there is some personal investment in the alliance. Participants from four age groups - pre-pubertal (8-10 years), early adolescents (12-14 years), late adolescents (16-17 years) and young 

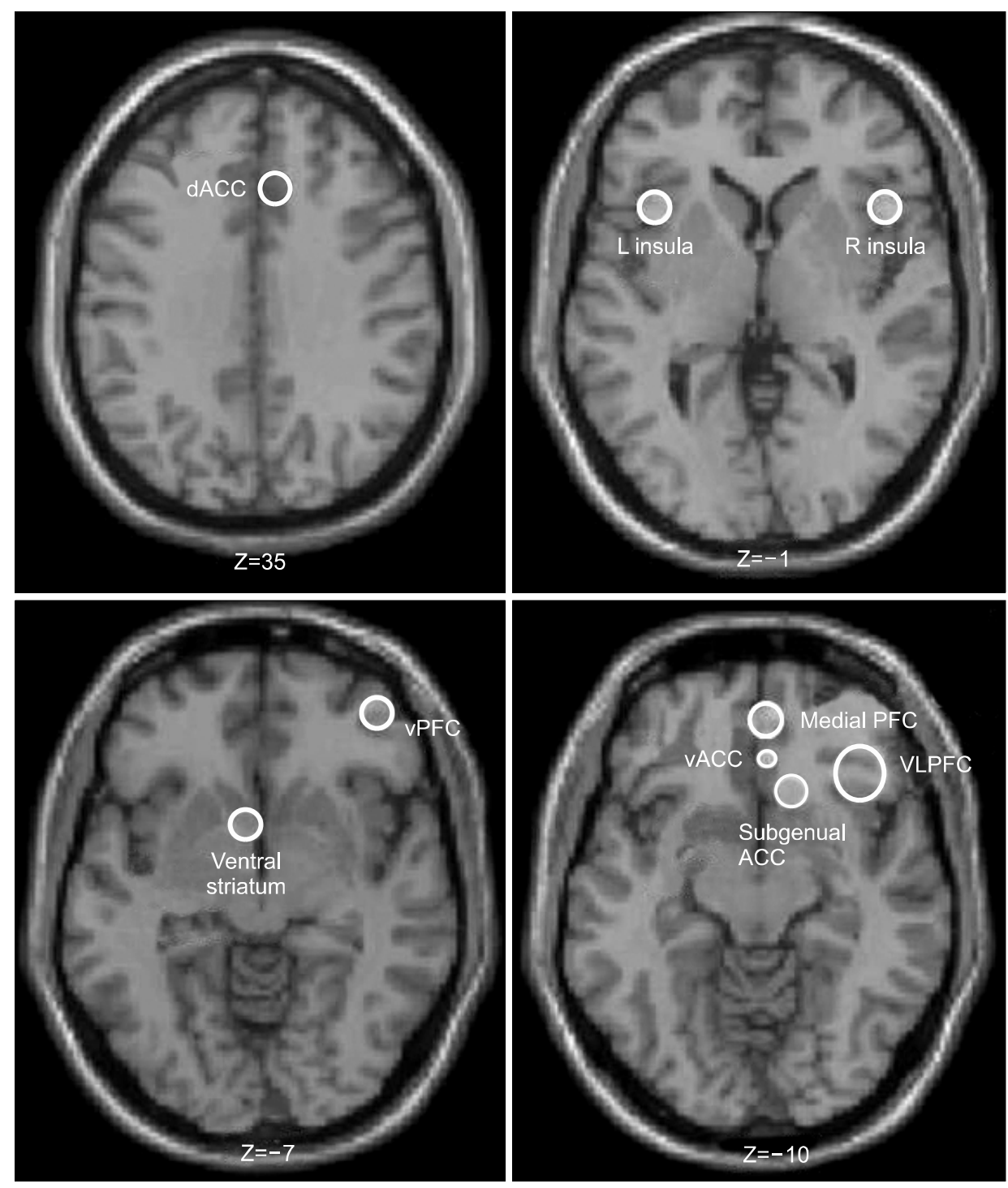

Fig. 2. Regions of interest activated or deactivated during the rejection task correlated that were correlated with different self- report measures across studies. The $\mathrm{MNI}$ coordinates from each study were averaged (see Table 2). The slices are in the axial view and the size of the ring represents the variability around the $y$-axis. ACC anterior cingulate cortex; $\mathrm{dACC}$, dorsal ACC; L Insula, left insula PFC, prefrontal cortex; PCC, posterior cingulate cortex; $R$ Insula right insula; $V A C C$, ventral $A C C$; VLPFC, ventrolateral PFC; VPFC, ventral PFC.

adults (19-25 years) - performed the social feedback task. When the expectation to be liked was followed by feedback of being liked (yes-yes), the right subcallosal cortex/ventral medial PFC was activated more than when the expectation to be liked was followed by feedback to not be liked (yes-no) in all groups except the pre-pubertal group, although no linear increase with age group in activity in this region or any other region was observed for this comparison. The reverse contrast - when the expectation to be disliked was followed by feedback of being disliked (no-no) compared to when the expectation to be disliked was followed by feedback of being liked (no-yes) - yielded a linear increase in activity in age in the left subcallosal cortex, bilateral orbitofrontal gyrus, left paracingulate cortex, left putamen, right insula, right parahippocampal gyrus and bilateral fusiform gyrus. Thus, consistent with the findings by Somerville et al., ${ }^{13)}$ the ventral medial PFC is involved in social evaluation particularly when the feedback is positive, but the present study additionally found that this response occurred when feedback was contingent on the participant's preference towards the other person being positive. The absence of this neural response in pre-pubertal adolescents may suggests that such social evaluation may be less defined in this age group, as participants in this age group also tended to judge pictures more quickly than did the adults.

Somerville et $_{\text {al }}{ }^{23)}$ examined the role of self-esteem on the neural response to positive or negative feedback (like or dislike the participant) received from others after participants chose whether they liked the pictured person or not or believed that the pictured person would like them or not as described above. After scanning, participants 
$(n=44$, mean age $=20$ years; and study 2 where the participant decided whether the other person would like the participant or not, $\mathrm{n}=22$, mean age $=19$ years) completed questionnaires on self-esteem, state and trait anxiety, rejection sensitivity and personality. Congruence (congruent: both participant and other rating each other as liked or disliked, incongruent: participant and other had opposite rating) by feedback (positive, negative) by self-esteem (high, low based on median split) interactions in a priori hypothesized regions of interest, region of interest (dACC, subgenual ACC/medial PFC) were examined. The feedback-by-self-esteem interaction in left subgenual ACC/medial PFC was significant, such that low self-esteem individuals showed heightened activation to positive feedback and weaker activation to negative feedback. High self-esteem individuals showed comparable responses to both types of feedback. A regression of self-esteem scores on the whole brain for the positive vs. negative feedback revealed activation clusters in left subgenual ACC and right medial PFC, but also right thalamus. These neural responses may relate to the need for general approval from others and maintaining one's social status (to be seen to be liked by others), rather than experience of rejection where the person is involved in a social transaction in which the person expresses him/herself favourably that prompts the other person(s) to exclude them. This may explain why a correlation was not found between the subgenual ACC/medial PFC response and rejection sensitivity. It may be that those with high self-esteem also have high social competence and tend to respond to negative social feedback received from others on par with positive feedback in order to maintain their social desirability. Low self-esteem individuals may experience more ambivalence about mixed responses.

Overall, the findings suggest that the subgenual $\mathrm{ACC} /$ medial PFC is involveed in receiving positive feedback from peers. However, no conclusive observations were made regarding receiving negative feedback from peers when participants expressed positive social preference of the peers. These findings based on the social evaluation task are in stark contrast to those based on Cyberball where dACC response is typically observed during exclusion. The dACC response may occur when there is more direct rejection as a result of expectancy violation. ${ }^{11,20)}$ The incongruence between participant preference and peer feedback in the social evaluation task, even though a more direct test of rejection during social interaction, may not be as sensitive a measure of rejection compared to the Cyberball task.

\section{REJECTION CUES AS WORDS OR PICTURES}

Kross et al. ${ }^{15)}$ presented paintings depicting rejection and acceptance to individuals high and low ( $\mathrm{n}=10$ in each group, mean age $=24$ years) in rejection sensitivity. As a measure of distress, participants rated the pictures on arousal, interest, pleasantness and security while undergoing fMRI. All participants activated the dACC, right VLPFC and insula more during rejection than acceptance scenes, besides several other un-hypothesized areas, namely left posterior cingulate, bilateral middle frontal gyrus (BA 46), inferior frontal gyrus (BA 47), medial frontal gyrus (BA 6), precentral gyrus (BA 6) and bilateral parahippocampal gyrus. Of these three hypothesized regions, low rejection sensitive (RS) individuals activated the dACC and right VLPFC more than high RS individuals. Low RS individuals also activated the left inferior frontal gyrus and right superior frontal gyrus more than high RS individuals during rejection than acceptance conditions. Greater distress was associated with lower dACC activity and greater insula activity. These findings seem to suggest reversal of effects normally found associated with rejection in high $\mathrm{RS}$ individuals. It may be that presenting rejection stimuli where the RS participant is a passive viewer of rejecting scenes may evoke a defensive response (down-regulation), compared to when the participant is actively engaged in rejection possibly because RS individuals may distance themselves from these scenes.

This trend for a reversal of the neural response to pictures depicting rejecting social interaction was also observed in individuals with high level of schizotypal unusual experiences. ${ }^{16)}$ Schizotypy is a personality trait along the normal continuum characterised by odd beliefs, such as magical thinking and belief in paranormal experiences, as well as anhedonia. ${ }^{24)}$ In their study, Premkumar et al. $^{16)}$ drew a sample of individuals who were high $(n=12)$ or low $(n=14)$ on schizotypal unusual experiences (mean age 29 years). Participants viewed pictures of social interactions depicting rejection, acceptance and neutral scenes while undergoing fMRI. Participants rated the pictures on arousal, rejection and affect. Participants also rated their current mood, level of rejection sensitivity, depression and anxiety. Across the whole sample, wholebrain analyses during rejection compared to acceptance scenes revealed greater activation in the lingual gyrus that is known to respond to facial emotional expressions. Low schizotypy individuals showed greater dACC, right VLPFC and medial PFC activation during rejection than 
neutral scenes compared to high schizotypal individuals. The findings suggested down-regulation of centres for rejection experience and emotion regulation when high schizotypal participants were passive viewers of rejection scenes and that the high schizotypy group may be distancing themselves from the scenes in order to minimize distress. However, it seems contradictory that activation was lower in brain areas involved in emotion regulation, namely right VLPFC and medial PFC, if response to rejection scenes was being suppressed.

Presenting rejection cues as pictures may give greater opportunity for manipulation of degree of engagement with the stimuli than would tasks that involve the participant experiencing exclusion or negative feedback. In tasks using pictures, feelings of rejection may be induced indirectly through recognition of rejection in other people, thus posing a systematic difference in the way feelings of rejection are induced. Still, individuals with high rejection sensitivity or schizotypy may have altered ways of perceiving rejection in close relationships that is different from rejection due to social exclusion from peers. Exclusion from peers may reflect a need for affiliation with a larger social group, a situation that is of greater concern to adolescents in schools and other competitive environments. As Blackhart et al. ${ }^{25)}$ notes, 'exclusion is thus a broader, more encompassing term, insofar as it denotes all phenomena in which one person is put into a condition of being alone or is denied social contact. The difference lies principally in how specifically the excluded person has sought the connection.' Rejection in close relationships may be more enduring and require more conscious coping strategies.

\section{CONCLUSION}

Across the different paradigms of social rejection, there is some degree of correspondence between studies in dACC involvement (Table 1). dACC involvement in the social evaluation task may relate to conflict detection from a purely error monitoring stance such as incongruence between participant preference and feedback received, ${ }^{13)}$ whereas in the social exclusion task dACC involvement may relate to how painful the rejection experience is. ${ }^{11,17-19)}$ The monitoring of distress due to rejection is mirrored in tasks where rejection is depicted in scenes of social interaction. $^{15,16)}$ Subgenual ACC and ventral ACC are varyingly involved in experiencing rejection, perhaps in compensation for dACC involvement in adolescents. ${ }^{20,21)}$

The medial PFC and VLPFC are instead involved in down-regulating the intensity of distress due to rejection as exemplified by their inverse association between activation in these regions and $\mathrm{AACC}$ response ${ }^{17,18)}$ and increased activation when exclusion is accompanied by social support, ${ }^{19)}$ but also their inverse association with distress. ${ }^{17,19)}$ The medial PFC also responds to receiving positive affective feedback from others ${ }^{13,22,23)}$ contingent on the individual's level of self-esteem ${ }^{23)}$ and therefore may be associated with the need for affiliation, rather than fear of rejection. Empathising with rejection leads to uniform down-regulation of dACC, VLPFC and medial PFC in individuals with a high level of rejection sensitivity ${ }^{15)}$ and schizotypy. ${ }^{16)}$ Under such conditions, participants may employ avoidance strategies, such as distancing themselves from the scenes. Further research is needed into understanding the nature of avoidance as a coping strategy when individuals are directly confronted with rejection during fMRI either as social exclusion or in close relationships, such as in the form of verbal criticism.

\section{REFERENCES}

1. Rosenfeld HM. Social choice conceived as a level of aspiration. J Abnorm Psychol 1964;68:491-499.

2. Mehrabian A, Ksionzky S. Models for affiliative and conformity behavior. Psychol Bull 1970;74:110-126.

3. Downey G, Feldman SI. Implications of rejection sensitivity for intimate relationships. J Pers Soc Psychol 1996;70: 1327-1343.

4. Caputi M, Lecce S, Pagnin A, Banerjee R. Longitudinal effects of theory of mind on later peer relations: the role of prosocial behavior. Dev Psychol 2012;48:257-270.

5. Lemay EP, Clark MS. "Walking on eggshells": how expressing relationship insecurities perpetuates them. J Pers Soc Psychol 2008;95:420-441.

6. Eisenberger NI. The pain of social disconnection: examining the shared neural underpinnings of physical and social pain. Nat Rev Neurosci 2012;13:421-434.

7. Carter CS, van Veen V. Anterior cingulate cortex and conflict detection: an update of theory and data. Cogn Affect Behav Neurosci 2007;7:367-379.

8. Walton ME, Croxson PL, Behrens TE, Kennerley SW, Rushworth MF. Adaptive decision making and value in the anterior cingulate cortex. Neuroimage 2007;36(Suppl 2):T142-T154.

9. Langens TA, Schüler J. Written emotional expression and emotional well-being: the moderating role of fear of rejection. Pers Soc Psychol Bull 2005;31:818-830.

10. Sinclair HC, Ladny RT, Lyndon AE. Adding insult to injury: effects of interpersonal rejection types, rejection sensitivity, and self-regulation on obsessive relational intrusion. Aggress Behav 2011;37:503-520.

11. Eisenberger NI, Lieberman MD, Williams KD. Does rejection hurt? An FMRI study of social exclusion. Science 2003;302:290-292.

12. Tajfel H, Turner J. An integrative theory of intergroup relations. In: Austin WG, Worchel $S$, editors. The social psychology of intergroup relations. Monterey:Brooks/Cole;1979. p.33-47.

13. Somerville LH, Heatherton TF, Kelley WM. Anterior 
cingulate cortex responds differentially to expectancy violation and social rejection. Nat Neurosci 2006;9:1007-1008.

14. Nesdale D, Duffy A. Social identity, peer group rejection, and young children's reactive, displaced, and proactive aggression. Br J Dev Psychol 2011;29:823-841.

15. Kross E, Egner T, Ochsner K, Hirsch J, Downey G. Neural dynamics of rejection sensitivity. J Cogn Neurosci 2007;19: 945-956.

16. Premkumar P, Ettinger U, Inchley-Mort S, Sumich A, Williams SC, Kuipers E, et al. Neural processing of social rejection: the role of schizotypal personality traits. Hum Brain Mapp 2012;33:695-706.

17. Eisenberger NI, Taylor SE, Gable SL, Hilmert CJ, Lieberman MD. Neural pathways link social support attenuated neuroendocrine stress responses. Neuroimage 2007;35:1601-1612.

18. Onoda K, Okamoto Y, Nakashima K, Nittono H, Ura M, Yamawaki S. Decreased ventral anterior cingulate cortex activity is associated with reduced social pain during emotional support. Soc Neurosci 2009;4:443-454.

19. Onoda K, Okamoto Y, Nakashima K, Nittono H, Yoshimura $\mathrm{S}$, Yamawaki S, et al. Does low self-esteem enhance social pain? The relationship between trait self-esteem and anterior cingulate cortex activation induced by ostracism. Soc Cogn
Affect Neurosci 2010;5:385-391.

20. Masten CL, Eisenberger NI, Borofsky LA, Pfeifer JH, McNealy K, Mazziotta JC, et al. Neural correlates of social exclusion during adolescence: understanding the distress of peer rejection. Soc Cogn Affect Neurosci 2009;4:143-157.

21. Sebastian CL, Tan GC, Roiser JP, Viding E, Dumontheil I, Blakemore SJ. Developmental influences on the neural bases of responses to social rejection: implications of social neuroscience for education. Neuroimage 2011;57:686-694.

22. Gunther Moor B, van Leijenhorst L, Rombouts SA, Crone EA, Van der Molen MW. Do you like me? Neural correlates of social evaluation and developmental trajectories. Soc Neurosci 2010;5:461-482.

23. Somerville LH, Kelley WM, Heatherton TF. Self-esteem modulates medial prefrontal cortical responses to evaluative social feedback. Cereb Cortex 2010;20:3005-3013.

24. Vollema MG, van den Bosch RJ. The multidimensionality of schizotypy. Schizophr Bull 1995;21:19-31.

25. Blackhart GC, Nelson BC, Knowles ML, Baumeister RF. Rejection elicits emotional reactions but neither causes immediate distress nor lowers self-esteem: a meta-analytic review of 192 studies on social exclusion. Pers Soc Psychol Rev 2009;13:269-309. 